\title{
Simulation of the dust suppression process with foam in the areas of belt conveyors
}

\author{
Vadim Bespalov ${ }^{1}$, Oksana Gurova ${ }^{1}$, Natalia Samarskaya ${ }^{1,}{ }^{*}$, and Ekaterina Lysova ${ }^{1}$ \\ ${ }^{1}$ Architectural and Construction Academy Don State Technical University, Sotsialisticheskaya str. \\ 162, Rostov-on-Don, 344022, Russia
}

\begin{abstract}
The article provides the analysis of the physical essence and simulation of the process of dust retention with foam in the air of working zones of belt conveyors transporting sand, crushed stone, gravel, coal, grain. In accordance with the proposed physical-energy concept of simulation of the process of dust control its physical essence is in a deliberate sequential action on the dust particles with previously prepared by the parameters external (additional) dispersed systems. Use of dust retention technology foam method provides high efficiency of reducing the concentration of dust in the air of working areas of belt conveyors, which varies in the range of 85.0-99.0\%, which provides the standard level of dust contamination (MPC) in air of working areas of listed industrial sources of formation and emission of dust.
\end{abstract}

\section{Introduction}

In accordance with the notions of the proposed physical and energetic concept for simulation of the process of dust suppression its physical essence is in purposeful consecutive impact on dust particles created, produced from the volume of technological raw material and spread in the air of internal and external working zones by prepared in advance as to the parameters external ("additional") disperse systems. Such a consequence of influence in practice is realized in the following stages: dust particles bunching, their interception, particulate control, air purification from dust particles and their dispersion in surface layer of the atmosphere [1-8].

One of the main stages of this process providing for regulatory level of dust pollution (LDP) in the air of working zones is dust interception close to the main stream of technological raw material in the process of its manufacturing or processing. The most often used in practice technologies of dust interception realization process are as follows:

- aerodynamic method by linear or swirling boost air flows;

- hydro-dynamic method by low-pressure irrigation;

- hydro-dynamic method by foam way;

- mechanical method by solid rigid body (by discreet solid bodies).

Out of the listed methods the foam one has a number of advantages: minimum fluid consumption, big surface of interphase interaction of dispersed fluid and dust particles,

\footnotetext{
*Corresponding author: nat-samars@yandex.ru
} 
prevention of over moisturizing of technological raw material, possibility of efficient use on mobile sources of dust liberation and formation, and also under the conditions of negative temperatures of air environment, which, in the end, provides for the possibility of considerable increase of efficiency of dust suppression, especially of finely-divided, most pneumonoconiosis-dangerous dust fractions [6, 9-12]. As a rule, foam method is used for spread sources of dust release (different types of transporters: belt, bucket, auger-type).

With the purpose of adherence to regulatory values of critical concentration of dust in the air of working zones of spill belt conveyors it is necessary to choose different technical means for protection of air media at the enterprises. This choice is related to carrying out of quality simulation of the efficiency of the process for decrease of dust concentration in the air of working zones of spill belt conveyors based on foam dust interception.

For manufacturing conditions of industrial enterprises, first of all, of enterprises of machine-building sphere, mathematical dependencies have been obtained, describing main parameters of foam and dust aerosol on the stages of dust interception on basis of revealing of interconnection between energetic parameters of the process of de-dusting and physical and chemical parameters of foam.

Studying of dust aerosol as a disperse system, behavior of dust particles under the conditions of external effects, examination of a number of tasks related to decrease of dust concentration in the air, improvement of the de-dusting technique have been reflected in the works of Azarov V.N., Amekhanov R.A., Boguslavskiy E.I., V.V., Zhuravlev V.K., Zhuravlev V.P., Zaburdyaev G.S., Ishchuk I.G., Kirin B.F., Klebanov F.S. Lifchak I.F., Logachev I.N., Lugovskiy S.I., Lujyanov A.B., Menkovskiy M.A., Minko V.A., Nikitin V.S., Saranchuk V.I., Sidorenko V.F., Strakhova N.A., Khezhev T.A., Shtockman E.A. And many other authors.

\section{Materials and methods}

For optimal realization of the process of dust interception it is necessary to provide for quality simulation of the process, using efficiency of dust suppression as the resulting parameter of the process, preliminary giving its mathematical description. In these works the underlying concepts of the mechanics of multi-phase media, aerodynamics, thermodynamics, physical and colloid chemistry, main principles of the technology of realization of air de-dusting in the complex with the results of experiments held with the use of high precision measuring devices have been used.

\section{Results}

For simulation of the process of decrease of dust concentration in the air of working zones of spill belt conveyors on basis of foam dust interception it is necessary to determine in each case of its practical application the corresponding resulting parameters, which include, first of all, efficiency of dust interception characterizing the decree of decrease of dust concentration in the air of working zone related to its initial concentration.

In accordance with the main provisions of the suggested by us physical and energetic concept for simulation of dust interception as one of the main stages for implementation of the process of dust suppression we have carried out mathematical description related to obtaining of parametrical dependence of the efficiency of dust suppression as the resulting parameter.

Description of the efficiency of dust interception is based on the consideration of probable realization of consecutive interdependent physical mechanisms of interaction of 
dust particles with certain bubbles of foam layer, which could be described with the following dependence:

$$
E_{e f f-d r}=1-\left(1-E_{e f f(1)-d r}\right) \cdot\left(1-E_{e f f(2)-d r}\right) \cdot\left(1-E_{e f f(3)-d r}\right)
$$

where $E_{\text {eff }(1)-d r}$ - is the efficiency of capture and return of dust particles by the foam layer into the stream of technological raw material under the influence of inertia forces; $E_{\text {eff(2)-dr }}-$ is the efficiency of elastic interaction (repulsion) of dust particles with foam bubbles; $E_{\text {eff( }(3)-}$ $d r-$ is the efficiency of capture of dust particles by foam bubbles under the influence of electric forces.

Thus, efficiency $E_{\text {eff( }(1)-d r}$ characterizes capture and return of dust particles by foam layer into the stream of technological raw material on the first stage on interaction under the influence of inertia forces and depends on the coefficient of gravity sedimentation.

Process of shielding of dust particles with the foam layer is characterized with efficiency $E_{\text {eff(2)-dr }}$, the value of which depends, mostly, on properties of foam and speed of encounter of dust particles with foam bubbles. Taking into account the results of experimental and theoretical research, represented in works [13-14], applied to foam layer the value of $E_{\text {eff(2)-dr }}$ is determined by the formula:

$$
E_{e f f(2)-d r}=1-\exp \left[-0,693 \cdot\left(v_{c r}^{\ni} / v\right)^{2}\right]
$$

where $v^{{ }}{ }_{c r}$ - is the critical speed of a dust particle, at which foam bubble could be destroyed at, $\mathrm{m} / \mathrm{s}$.

Capture of dust particles by foam under the influence of electric forces [15-16] is characterized by efficiency $E_{\text {eff(3)-dr }}$. Analysis of experimental data [8] allowed to obtain the dependence of efficiency of capture of dust particles under the influence of electrical forces which we use in calculation in relation to foam method in using foam layer:

$$
E_{e f f(3)-d r}=1,7 \cdot 10^{-3} \cdot q_{0}^{0,65}
$$

where $q_{0}-$ is electrical mass per charge unit of foam, $\mathrm{Kl} / \mathrm{m}$.

It should be noted that for artificial electrilization the value of electrical mass per charge unit $q_{0}$ depends on the method of foam recharging and potential value [1].

Taking into consideration equations (2) and (3) dependence (1) of the efficiency of dust interception by foam method using foam layer takes the following form:

$$
\begin{aligned}
& E_{e f f-d r}=1-d_{p} \cdot v \cdot \rho_{p} /\left(d_{p} \cdot v \cdot \rho_{p}+360 \cdot \mu_{o} \cdot l\right) . \\
& \cdot \exp \left[-7,48 \sigma / v^{2} \cdot \rho_{p} \cdot\left(l / d_{p}+2,1 d_{p} / d_{n} \cdot\left(d_{p}+4 \cdot 10^{-7}\right)\right)\right] . \\
& \cdot\left(1-1,7 \cdot 10^{-3} \cdot q^{0,65}\right)
\end{aligned}
$$

Thus, we have obtained parametric dependence of efficiency (4) for the process of dust interception based on application of foam layer as its resulting parameter, allowing to simulate the result of implementation of this process taking into account adherence to sanitary and hygienic and ecological norms.

\section{Discussion}

Technologically foam method of dust interception is implemented so that close to the surface (volume) of technological raw material a foam layer is formed in the form of 
protection shield representing an "additional" disperse system, preventing dust particles liberation into the air of working zones and providing for return of these particles into the technological raw material due to adhesion forces, preventing their secondary pick-up. At that we suggest that dust particles in this case shall be reviewed from the positions of the theory of disperse systems, taking into account surface peculiarities of the dust aerosol.

Hydrodynamic method of dust interception by foam method is implemented in accordance with technological scheme represented in the fig. 1. At that foam layer shall be used with elastic shielding properties (“dry foam”).

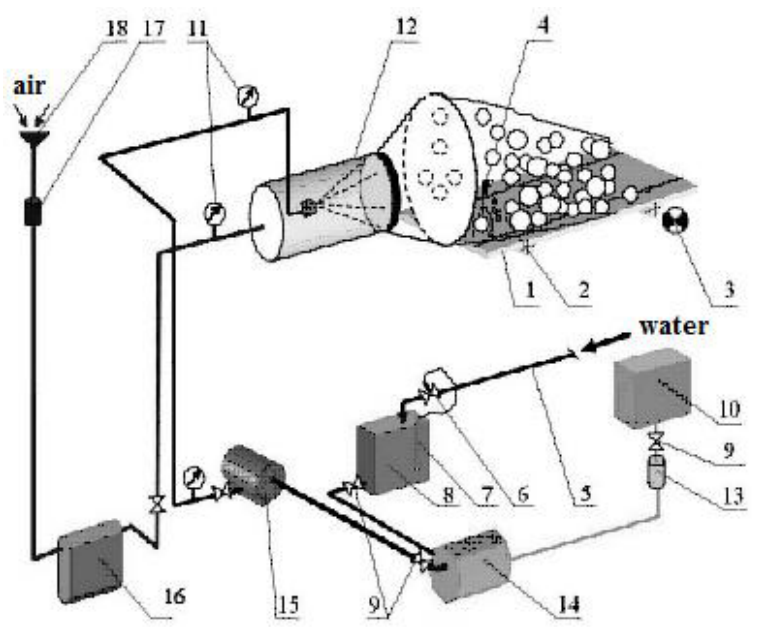

Fig. 1. Flow chart of hydrodynamic method of DP interception with foam. 1- portion of belt conveyor (mobile process equipment); 2- source of dust particles; 3-working zone; 4- discharged dust particles; 5- water pipe main line; 6- magnetic valve; 7- medium level regulator; 8- source water tank; 9- shutoff and control valves; 10 - tank for foaming agent storage; 11- manometer; 12- netted foam generator; 13- metering unit; 14- mixer; 15- pumping system; 16 - ventilator; 17- air filter; 18 - air-intake device

The principle of the flow chart is as follows. Water from the main line 5 through the magnetic valve 6 comes into tank 8, the level of fluid in which is supported with the help of medium level regulator 7. From the tank 8 with the help of pumping system 15 water comes to mixer 14. At that from the tank 10 foaming agent through the metering unit 13 also comes to mixer 14, in which the foaming agent solution is formed with the set concentration, evenly distributed in the fluid. The ready solution of foaming agent with the help of pumping system 15 is fed to net of foam generator 12. At that surrounding air through the air-intake device 18 is taken by the ventilator 16 and through filter 17 is also fed to the net of foam generator 12, in the process of operation of which a foam layer is produced directed to the surface of the source 2 of dust formation, creating a shielding protective layer.

\section{Conclusions}

Practical implementation of the technology of dust interception by foam method on belt conveyors of enterprises of various spheres of industry transporting burnt molding sand, sand, crushed rock, gravel, coal carried out by us on basis of numerical simulation has shown that, first of all, calculated values of efficiency of decrease of dust concentration in the air of working zones correspond to its actual values within the error limit $\pm 2.5 \%$ with 
confidential probability 0.95 , and, secondly, that for this type of technological equipment rather high values of efficiency are provided within the range from 85.0-99.0\%, which allows to reach regulatory levels of dust pollution (LDP) in the air of working zones of the listed industrial sources for creation and distribution of dust.

\section{References}

1. V. I. Bespalov, O. S. Gurova, N. S. Samarskaya, Biosciences Biotechnology Research Asia, 12 (2015)

2. W. Cheng, Y. Ma, J. Yang, B. Sun, International Journal of Mining Science and Technology, 26, 1025-1032 (2016)

3. E. Y. Kozlovtseva, V. F. Loboyko, D. A. Nikolenko, Procedia Engineering, 150, 19541959 (2016)

4. O. S. Gurova, Scientific review, 24, 68-71 (2015)

5. B. Górka-Kostrubiec, Building and Environment, 90, 186-195 (2015)

6. V. I. Bespalov, O. S. Gurova, N. S. Samarskaya, E. P. Lysova, A. N. Mishchenko, Biosciences Biotechnology Research Asia, 11, 1615-1620 (2014)

7. J. Richards, R. Reif, Y. Luo, J. Gan, Environmental Pollution, 214, 290-298 (2016)

8. R. A. Ogle, Dust Explosion Dynamics, 211-305 (2017)

9. Q. Li, K. Wang, Y. Zheng, M. Ruan, B. Lin, Powder Technology, 292, 290-297 (2016)

10. Z. Wang, Science of The Total Environment, 553, 673-675 (2016)

11. A. V. Azarov, N. S. Zhukova, E. A. Kalyuzhina, Procedia Engineering, 150, 20802086 (2016)

12. V. Azarov, M. Trokhimchyk, O. Sidelnikova, Procedia Engineering, 150, 2008-2012 (2016)

13. X. Wei, B. Gao, P. Wang, H. Zhou, J. Lu, Ecotoxicology and Environmental Safety, 112, 186-192 (2015)

14. D. Prostański, Journal of Sustainable Mining, 14, 108-114 (2015)

15. J. T. Salin, M. Salkinoja-Salonen, P. J. Salin, K. Nelo, H. Syrjälä, Environmental Research, 154, 234-239 (2017)

16. S. G. Davis, P. C. Hinze, O. R. Hansen, K. Wingerden, Journal of Loss Prevention in the Process Industries, 24, 837-846 (2011) 